\title{
Improving passive microwave sea ice concentration algorithms for coastal areas: applications to the Baltic Sea
}

\author{
By NINA MAA $\beta^{*}$ and LARS KALESCHKE, Institute of Oceanography, University of Hamburg, \\ 20146 Hamburg, Germany
}

(Manuscript received 5 August 2009; in final form 26 March 2010)

\begin{abstract}
Sea ice concentration can be retrieved from passive microwave data using the NASA Team algorithm or the Artist Sea Ice (ASI) algorithm, for example. The brightness temperature measurements obtained from the Special Sensor Microwave Imager (SSM/I) instrument or the Advanced Microwave Scanning Radiometer-EOS (AMSR-E) are commonly used for this purpose. Due to the coarse resolution of these instruments considerable systematic ice concentration errors in coastal regions occur. In the vicinity of the coast the instrument footprints usually contain both land and sea surfaces. Compared to sea surfaces, land surfaces are characterized by higher emissivities and lower polarization differences at the involved microwave channels. Thus, a systematic overestimation of coastal ice concentration is caused. In this paper, a method is developed to remove the land impact on the observed radiation. Combining a high-resolution data set for the shoreline and the antenna gain function the brightness temperature contribution originating from land surfaces can be identified. The brightness temperature related to the ocean fraction within the considered footprint can then be extracted. This separation technique is applied to SSM/I measurements in the Baltic Sea and the resulting ice concentration fields are compared to high-resolution satellite images. The highly complex shoreline of the Baltic Sea region provides an ideal area for testing the method. However, the presented approach can as well be applied to Arctic coastal regions. It is shown that the method considerably improves ice concentration retrieval in regions influenced by land surfaces without removing actually existing sea ice.
\end{abstract}

\section{Introduction}

Microwave sensor observation from satellite is particularly suitable for monitoring sea ice because it is independent from daylight and less affected by clouds and weather conditions. Moreover, the spatial and temporal coverage allow for continuous operational observation. However, the coarse resolution of passive microwave instruments causes considerable errors in coastal areas.

Drusch (2006) investigated the impact of different sea ice concentration data sets for the Baltic Sea on numerical weather prediction. The resulting sensible and latent heat fluxes differed significantly. Moreover, the differences in turbulent fluxes caused changes in the planetary boundary layer and thus affected the forecast cloud cover. The implementation of an improved ice concentration retrieval in the Baltic Sea where the land impact

\footnotetext{
* Corresponding author.

e-mail: nina.maass@zmaw.de

DOI: $10.1111 /$ j.1600-0870.2010.00452.x
}

on brightness temperatures is almost ubiquitous could therefore improve weather forecasts.

Sea ice algorithms such as the widespread NASA Team algorithm (Swift and Cavalieri, 1985) and the more recently developed Artist Sea Ice (ASI) algorithm (Spreen et al., 2008) are applied to retrieve ice concentrations from passive microwave data. Commonly, the brightness temperatures measured by the Special Sensor Microwave/Imager (SSM/I) or the Advanced Microwave Scanning Radiometer-EOS (AMSR-E) instrument are used for this purpose. Due to the large footprints associated with these sensors, the obtained ice concentration fields have very coarse resolutions.

The effect of the coarse resolution is particularly apparent near the shoreline. Brightness temperatures over land and ocean surfaces differ significantly. Where there is found both land and ocean surfaces within one footprint of the used channel a mixed brightness temperature signal is measured. The abovementioned ice concentration algorithms are restricted to ocean brightness temperatures. Thus, the generally higher emissivities above land surfaces compared to sea surfaces cause a systematic overestimation of ice concentration. 
The systematic retrieval errors caused by land spillover influence the comparability of different ice algorithms. Algorithms using lower resolved microwave data are more affected by land spillover than algorithms using more highly resolved data. Consequently, the low-resolution algorithms usually overestimate ice concentration relative to the high-resolution algorithms. Commonly, an enlarged land mask is used to avoid artificial algorithm performance deviations caused by the different resolutions (Maslanik et al., 1996). This approach is as well used by some operational services. It is very simple and effective, but ignores any information available from the coastal measurements.

An alternative method to overcome the difficulties in coastal ice concentration retrieval with passive microwaves is used by the National Snow and Ice Data Center (NSIDC). They use summer sea ice concentrations observed in the vicinity of coastlines to correct the retrieved ice concentration fields. This correction requires the presence of open water in the vicinity of the image pixel to be corrected and is in any case clearly a rough approximation. It does not take into account temporal variation of the land spillover effect.

We have developed an automated technique to remove the systematic error in coastal zones while exploiting the available information for coastal sea ice coverage in passive microwave data. Therefore, we extract the ocean contribution of any measured brightness temperature. This modified value is then inserted in ice concentration algorithms. To test our method it was applied to SSM/I data in the Baltic Sea and compared to higher resolution satellite images.

Due to its complex shoreline and archipelago, the Baltic Sea region provides a challenging field of application. On the one hand it would clearly benefit from an improved ice concentration retrieval in land-influenced areas. On the other hand it serves as an ideal area for testing the method. The presented approach can as well be used to improve ice concentration retrieval in Arctic coastal regions. In addition, applications to the Antarctic Peninsula are conceivable, where cruise ships are interested in reliable reports for coastal sea ice, for example.

We describe the developed approach for brightness temperature data obtained from the SSM/I instruments. When modifications for the antenna gain are embedded, the method can also be adjusted to the other passive microwave sensors such as the Special Sensor Microwave Imager/Sounder (SSMI/S) instrument on the Defense Meteorological Satellite Program (DMSP) F17-platform, AMSR-E on Aqua satellite and the Microwave Imager/Sounder (MIS) on the National Polar-orbiting Operational Environmental Satellite (NPOESS) which is planned to be launched in 2016 .

The paper is structured as follows. After a short description of the data used in this study, we introduce the ice concentration algorithms we used to test our separation method. Subsequently, we describe in more detail the land spillover problem in coastal areas and the previous methods to deal with it. In Section 4, we start with considering the technique of Bellerby et al. (1998) for separating land and sea brightness temperatures. We address the difficulties arising when this technique is applied to coastal areas with spatially variable sea ice cover. In the following, we present a new method adjusted to the requirements of coastal sea ice retrieval and consider different realizations of the basic idea. In Section 5, the SSM/I data corrected for land spillover are used to calculate ice concentration fields in the Sea of Bothnia. The results are compared to higher resolution satellite images. Finally, we discuss the results and identify error sources.

\section{Data set}

We applied the developed separation method to brightness temperatures measured by the SSM/I instrument. The SSM/I sensor measures the radiation emitted by the Earth's surface at 19.35, 22.235, 37.0 and $85.5 \mathrm{GHz}$ (Hollinger, 1990). At 22.235 GHz, only the vertically polarized radiation is measured, at the remaining frequencies both vertical $(\mathrm{V})$ and horizontal $(\mathrm{H})$ polarization are measured. To simplify matters these channels will be referred to as the $19 \mathrm{~V}, 19 \mathrm{H}, 22 \mathrm{~V}, 37 \mathrm{~V}, 37 \mathrm{H}, 85 \mathrm{~V}$ and $85 \mathrm{H}$ channels in the following.

We used the data recorded by the SSM/I instrument carried on board the DMSP F13 platform. The instrument rotates at a uniform rate of $31.6 \mathrm{rpm}$ and records the radiation measured over an angle of $102.4^{\circ}$ in forward direction. Contemporaneously, the instrument track moves at $6.58 \mathrm{~km} \mathrm{~s}^{-1}$ along the Earth's surface. The sampling interval of the rotating radiometer at $85 \mathrm{GHz}$ is half the time interval for the lower frequencies, which are sampled every $8.44 \mathrm{~ms}$.

Due to the superposition of the sensor rotation and the satellite forward motion sample spacing in cross-track direction and as well along the in-track direction is $12.5 \mathrm{~km}$ for the $85 \mathrm{GHz}$ channels and $25 \mathrm{~km}$ for the remaining channels.

Every measured brightness temperature value results from the radiation emitted by an area on the Earth's surface modified by the overlying atmosphere. The instantaneous field of view (IFOV) is the projection of the antenna $-3 \mathrm{~dB}$ beam width onto the Earth's surface. The $-3 \mathrm{~dB}$ distance is defined by a decrease of antenna gain to half of its value compared to the centre point of the footprint. The IFOV of the SSM/I instrument is approximately elliptical.

The effective field of view (EFOV) is obtained taking into account the integration time of the radiometer. The EFOV is also assumed to be of elliptic form. The along-track and crosstrack sizes of the EFOV represent the corresponding major and minor axes (Table 1).

A schematic view of the SSM/I footprint distribution is shown in Fig. 1.

The separation method requires accurate knowledge about the land-water distribution within the field of application. We used the Generic Mapping Tools (GMT) to create a land mask from the Global Self-consistent, Hierarchical, High-resolution Shoreline Database (GSHHS) (Wessel and Smith, 1996). The 
Table 1. SSM/I channel sample characteristics

\begin{tabular}{lcccc}
\hline Channel & $19 \mathrm{~V} / 19 \mathrm{H}$ & $22 \mathrm{~V}$ & $37 \mathrm{~V} / 37 \mathrm{H}$ & $85 \mathrm{~V} / 85 \mathrm{H}$ \\
\hline$-3 \mathrm{~dB}$ Footprint $(\mathrm{km} \times \mathrm{km})$ & $69 \times 43$ & $60 \times 40$ & $37 \times 28 / 37 \times 29$ & $15 \times 13$ \\
Sample resolution $(\mathrm{km} \times \mathrm{km})$ & $25 \times 25$ & $25 \times 25$ & $25 \times 25$ & $12.5 \times 12.5$ \\
\hline
\end{tabular}

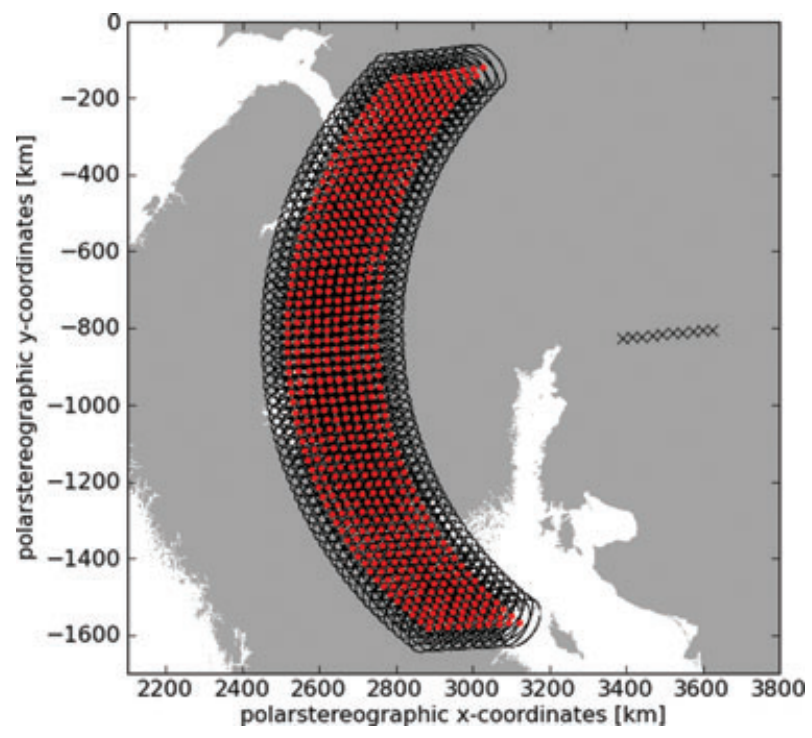

Fig. 1. A schematic view of the SSM/I footprint scan geometry. Although the platform is moving the SSM/I instrument is rotating and recording data within an angle of $102.4^{\circ}$ in the forward direction. In the figure the centre points and the surrounding footprints of the $19 \mathrm{GHz}$ channels are depicted for a couple of successive scans.

GSHHS shorelines are constructed from hierarchically arranged closed polygons. The mean point separation of data points is $178 \mathrm{~m}$. However, the point separation is locally variable and ranges from a few meters to a maximum of $24 \mathrm{~km}$ in Antarctica. We assume that the Baltic Sea region is highly resolved.

For calculations and the depiction of our results we use the polar-stereographic projection as used, for example by the NSIDC (National Snow and Ice Data Center). This projection specifies a projection plane to the Earth's surface at $70^{\circ}$ northern and southern latitude. This approach causes no distortion at $70^{\circ}$ latitude yielding minimum distortion at the marginal ice zone. Distortion increases with increasing distance from the $70^{\circ}$ circle of latitude but amounts to only $7 \%$ at the poles and is therefore particularly suitable for polar applications.

To evaluate our method we compared the resulting ice concentration fields to Advanced Synthetic Aperture Radar (ASAR) and MODerate resolution Imaging Spectroradiometer (MODIS) images.

The ASAR sensor is an active microwave sensor operating at $5.331 \mathrm{GHz}$ (C-band). It is mounted on board the ENVironmental SATellite (ENVISAT). ASAR measurements are independent of daylight and clouds. The measured backscatter signatures of sea ice and wind-induced rough ocean surfaces are often similar and thus hard to discriminate. In this study, we used Wide Swath Mode ASAR images with a resolution of $150 \mathrm{~m} \times 150 \mathrm{~m}$.

MODIS sensors are passive sensors measuring radiation reflected and/or emitted from the target in the visible and infrared spectrum. Sea ice measurements are constrained to cloud-free daytime observations. In this study, we used the reflectivities measured at MODIS band 1 by the instrument mounted on the Terra platform. Band 1 measurements have a spatial resolution of $250 \mathrm{~m} \times 250 \mathrm{~m}$ and include radiation in the wavelength range between 620 and $670 \mathrm{~nm}$, which is in the visible light spectrum.

\section{Sea ice algorithms}

\subsection{NASA Team algorithm}

The NASA Team algorithm uses the $19 \mathrm{~V}, 19 \mathrm{H}$ and $37 \mathrm{~V}$ channels to retrieve sea ice concentration from passive microwave data (Swift and Cavalieri, 1985). Ice concentrations for multi- and first-year ice are computed separately. Their sum is the total ice concentration. They are computed from the polarization ratio (PR) at $19 \mathrm{GHz}$ and the spectral gradient ratio (GR) of the $19 \mathrm{~V}$ and $37 \mathrm{~V}$ channels. Therefore, brightness temperatures for the involved channels characteristic for open water, first-year ice and multi-year ice are required. For large-scale mapping of ice concentration a tie point set for the Arctic exists (Cavalieri et al., 1991). Steffen and Schweiger (1991) showed that regionally adjusted tie points improve the retrieval accuracy. Thus, we used the tie points Grandell and Hallikainen (1994) obtained for the northern Baltic Sea using SSM/I data. As the Baltic Sea is only seasonally ice covered, the multi-year ice tie point for the Arctic Ocean was maintained and only the total ice concentration is considered.

To reduce weather-related effects that cause spurious ice concentrations over open water areas, the NASA Team algorithm is usually used in conjunction with a weather filter. The weather filter and its adjustment to Baltic Sea conditions are discussed in Sections 3.3 and 5.2, respectively.

Sample spacing of the involved SSM/I channels is $25 \mathrm{~km} \times$ $25 \mathrm{~km}$. Spatial resolution of the retrieved ice concentration field is determined by the involved channel with the coarsest resolution. Using the NASA Team algorithm, spatial resolution of the ice concentration field is often declared to be $69 \mathrm{~km} \times 43 \mathrm{~km}$ according to the $-3 \mathrm{~dB}$ footprint size of the $19 \mathrm{GHz}$ channels. However, the actual footprint size accounting for most of the antenna gain is considerably larger. If $94 \%$ of the antenna gain 
is included, the effective resolution of the $19 \mathrm{GHz}$ channels will be $138 \mathrm{~km} \times 86 \mathrm{~km}$ (Drusch et al., 1999).

For the sake of simplicity we examined the NASA Team algorithm in this study. However, the approach described in the following can as well be applied to the more recent NASA Team 2 algorithm (Markus and Cavalieri, 2000).

\subsection{ASI algorithm}

The Artist Sea Ice (ASI) algorithm developed by Kaleschke et al. (2001) uses the $85 \mathrm{GHz}$ channels to retrieve sea ice concentration from SSM/I measurements. Total ice concentration is computed from the polarization difference $\mathrm{P}(85)$ at $85 \mathrm{GHz}$. The underlying idea is to exploit the fact that polarization differences observed over sea ice surfaces are much smaller than over open water. As $\mathrm{P}(85)$ is similar for all ice types, the ASI algorithm does not distinguish between multi-year ice and first-year ice. The retrieval requires pre-defined tie points for open water and $100 \%$ ice coverage. These tie points can vary regionally and seasonally depending on the variability of the atmospheric conditions (Spreen et al., 2008). We analysed SSM/I data from the winter seasons 2008 and 2009 to obtain optimal tie points for Baltic Sea conditions. The tie point selection is described in Section 5.1.

The pronounced atmospheric influence on brightness temperatures at $85 \mathrm{GHz}$ requires effective weather filters. They are described in Section 3.3.

Sample spacing of the $85 \mathrm{GHz}$ channels is $12.5 \mathrm{~km} \times 12.5 \mathrm{~km}$. Using the ASI algorithm spatial resolution of the ice concentration field is generally assumed to be $15 \mathrm{~km} \times 13 \mathrm{~km}$, although the effective resolution is again about twice as large.

\subsection{Weather filter}

Atmospheric effects may cause spurious ice concentrations to be assigned to actually ice free water areas using the NASA Team and ASI algorithm, respectively. The combination of two weather filters has been found to remove these spurious ice concentrations except for very few extreme weather events.

Effects caused by wind-induced surface roughening, cloud liquid water and rainfall are successfully eliminated by implementing a filter based on the gradient ratio GR(37V,19V) of the $37 \mathrm{~V}$ and $19 \mathrm{~V}$ channels (Gloersen and Cavalieri, 1986). The second filter primarily removes effects originating from high water vapour concentrations and ice temperature variations at the ice edge. For this purpose, the gradient ratio GR(22V, 19V) of the $22 \mathrm{~V}$ and $19 \mathrm{~V}$ channels is introduced (Cavalieri et al., 1995).

The influence of atmospheric cloud liquid water and water vapour on the measured brightness temperatures is particularly pronounced at $85 \mathrm{GHz}$ and thus for the ASI algorithm.

Spreen et al. (2008) give weather filter thresholds determined for Arctic applications. Sea surface salinity and thus bulk ice salinity in the brackish waters of the Baltic Sea are considerably lower than in the Arctic. According to Leppäranta and Myrberg (2009) water salinity in the upper layer of the Bay of Bothnia is $2-4$, the sea ice salinity being correspondingly lower. To adapt the gradient ratio thresholds to these conditions we analysed the gradient ratio values characteristic for open water and ice covered areas in the Sea of Bothnia. The threshold adaption is described in Section 5.2.

\subsection{Ice concentration retrieval in coastal regions}

The above-mentioned ice concentration algorithms are based on the emissivity differences between sea ice and ice-free sea surfaces at the involved microwave channels. The emissivity of first-year ice is higher than the emissivity of water for both polarizations at frequencies ranging between 5 and $100 \mathrm{GHz}$, including all SSM/I channels (Spreen et al., 2008). This is as well true for the emissivity of multi-year ice except for the vertically polarized 37 and $85 \mathrm{GHz}$ channels.

In comparison to open water surfaces, the land surfaces are also characterized by high emissivity values at the microwave spectra. The resulting average brightness temperature of an area containing both land and ice-free water surfaces is therefore in general increased compared to the value related to the pure ocean part of the footprint. Due to the closer similarity in emissivity between ice-covered ocean and land compared to ice-free ocean and land, the systematic brightness temperature deviation increases with decreasing ice concentration.

The application of both the NASA Team and the ASI algorithm to uncorrected passive microwave data in general leads to a considerable overestimation of ice concentration in the coastal regions. Actually ice-free coastal regions are assigned ice concentration values up to $100 \%$ closest to the coast, where the land impact is by far predominant. The ice concentration values decrease with increasing distance from the coast and consequently decreasing land impact (Figs. 6a, 6c, 8a and 8c).

In this context, the coastal region is defined as the region being distinctly affected by land surfaces. This definition includes not only the regions in the vicinity of the coastline but also ocean areas containing islands. If the footprints accounting for 99.9\% of the antenna gain are considered, all pixels containing both land and ocean surfaces within a distance defined by the threefold $-3 \mathrm{~dB}$-ellipse major axis will be classified to be coastal pixels. The region assumed to be coastal therefore depends on the footprint sizes of the SSM/I channels (see 1) used in each ice algorithm. For the NASA Team algorithm this region is within a distance of about $210 \mathrm{~km}(3 \times 69 \mathrm{~km})$ from any land surfaces. For the ASI algorithm the distance reduces to $45 \mathrm{~km}$ $(3 \times 15 \mathrm{~km})$.

The extent of erroneous ice concentration values related to the problem of brightness temperatures representing a mixture of land and water signatures has been investigated (NSIDC, 1996). In this analysis, SSM/I daily averaged brightness temperatures 
were used in conjunction with the Atmospheric Environment Service (AES)/York sea ice algorithm. The AES/York ice algorithm uses a linear combination of brightness temperatures from the 19 and $37 \mathrm{GHz}$ channels to retrieve ice concentration. One of the regional study areas was a rectangular box containing Scandinavia. Monthly averaged September sea ice concentrations from 1987 to 1994 were then analysed assuming ice-free conditions within the study area. All non-zero sea ice concentration values can thus be treated as erroneous values. The fraction of false sea ice concentration values was derived by dividing the number of pixels associated with false sea ice assignments by the total number of pixels representing ocean. Thus for the examined time period an average error rate of $30 \%$ was obtained for the Scandinavian region.

A common approach to deal with the land spillover is to enlarge the land mask to mask out coastal pixels. Maslanik et al. (1996) compared the ice extent as derived from the Scanning Multichannel Microwave Radiometer (SMMR) and the SSM/I using the NASA Team algorithm. Due to the SMMR instrument's lower resolution, the land impact on ice concentration retrieval is greater than for the concentration derived from SSM/I measurements. For the analysed period in summer 1987, the SMMR derived ice extent for the Northern Hemisphere map area used by the NSIDC products was found to be $12 \%$ greater than for SSM/I when the standard land mask was used. The ice extent is defined to be the summation of all grid cells with ice concentrations exceeding $15 \%$. Extending the land mask to cover $50 \mathrm{~km}$ of adjacent ocean led to a reduction of the difference in ice extent to $2.5 \%$ giving a rough estimation for the impact of land spillover on ice retrieval.

The NSIDC uses an extension of the method developed by Parkinson et al. (1987) to account for the problem of mixed ocean-land signatures. The approach is based on the assumption that a minimum observed sea ice concentration in the vicinity of coastlines where no sea ice remains offshore is the result of land spillover. The minimum values are generally observed in late summer. These minimum values are then subtracted from the original image. The subtraction causes errors in areas of actual sea ice cover. To reduce these errors open water pixels located in the vicinity of the image pixel to be corrected are required. The method can only be a rough approximation as it does not account for the fact that the amount of land spillover effects is temporally variable.

In the next section, an approach to overcome the land spillover problem in coastal regions is described.

\section{Separation method}

\subsection{Mixed pixel problem}

The brightness temperature $T_{\mathrm{B}}$ seen by the radiometer is the integral of the actual brightness temperature distribution within the elliptic observation area weighted with the antenna gain function

$T_{\mathrm{B}}\left(x_{0}, y_{0}\right)=\iint \bar{G}(x, y) T_{\mathrm{B}}(x, y) \mathrm{d} x \mathrm{~d} y$,

where $\bar{G}(x, y)$ is the normalized antenna gain function and $T_{\mathrm{B}}(x, y)$ is the actual brightness temperature at the position $(x, y)$. The integral is taken over the footprint ellipse.

The actual brightness temperature distribution results from a continuous field of radiation, where brightness temperatures above land and sea surfaces in general vary considerably. Bellerby et al. (1998) assumed that in a small region land and sea areas are characterized by two single independent brightness temperatures $T_{\text {land }}$ and $T_{\text {sea }}$, respectively. The brightness temperature measured by the satellite can then be expressed by

$T_{\mathrm{B}}=\alpha T_{\text {land }}+(1-\alpha) T_{\text {sea }}$,

where $\alpha$ is the convolution of the antenna gain function with the land-water-distribution associated with the considered footprint. The mathematical expression of $\alpha$ is

$\alpha\left(x_{0}, y_{0}\right)=\int \bar{G}(x, y) L(x, y) \mathrm{d} x \mathrm{~d} y$.

The land-water-distribution $L(x, y)$ is derived from a digitized land mask assigning the value one to land and zero to sea surfaces.

Bellerby et al. (1998) suggest that the nine grid points surrounding and including every measurement are examined. This approach benefits from the fact that adjacent SSM/I footprints are overlapping. Assuming uniform land and sea brightness temperatures an over specified system of linear equations can be established. This system consists of nine equations for the measured brightness temperature (eq. 2) and the two unknowns $T_{\text {land }}$ and $T_{\text {sea }}$. The brightness temperatures corresponding to land and sea areas, respectively, are obtained using least squares optimization.

For our purposes the separation technique by Bellerby et al. (1998) is applicable when the examined footprint's environment contains only two different surface types. This is for instance true when there is found either only land and ice-free sea surfaces or only land and completely sea ice-covered surfaces. In these cases, the assumption of two constant independent brightness temperatures for the land and sea areas is appropriate.

If the ice concentration influenced by land varies significantly, this assumption cannot be made without introducing large errors. To further illustrate this, we can consider an area under investigation containing land, open ocean and ice surfaces. A correspondent situation is found for the scan geometry at the fast ice surrounding the Swedish island Holmön (Fig. 2). The brightness temperatures associated with ice-free and ice-covered areas differ significantly. This distinction is the basic prerequisite for ice concentration retrieval using the NASA Team and ASI ice algorithms. Assuming a constant brightness temperature for the ocean part of the footprint is therefore not adequate. If this assumption is made nevertheless the obtained ocean brightness 


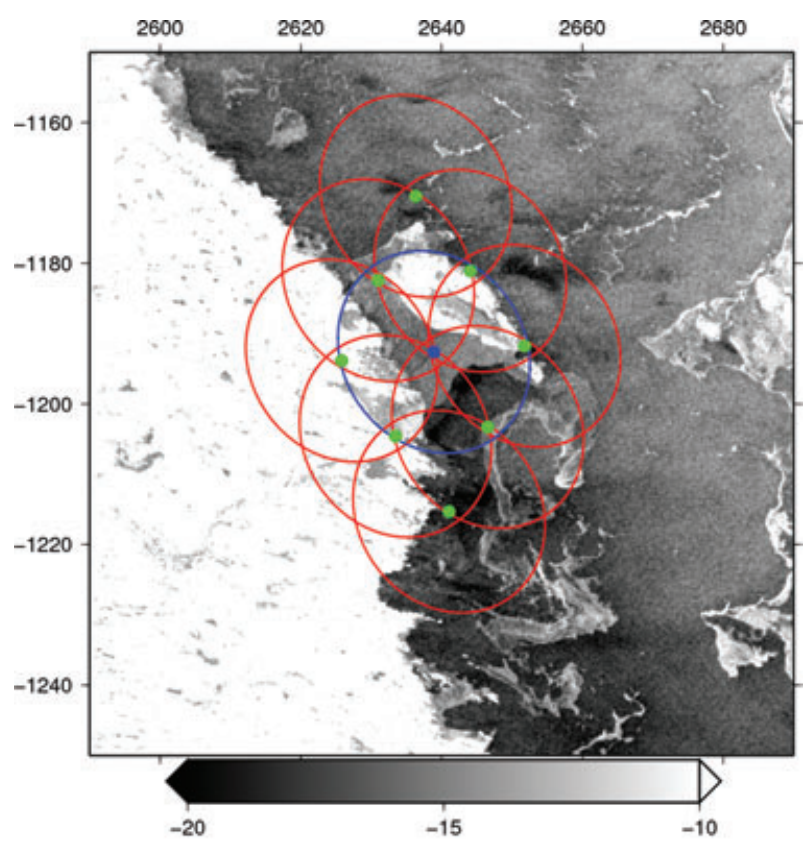

Normalized radar backscatter coefficient [dB]

Fig. 2. The centre points and the corresponding footprints of an exemplary $85 \mathrm{GHz}$ measurement and its $3 \times 3$ environment overlayed to an ASAR image. The image shows the ice situation in the vicinity of the Holmön island off the Swedish coast.

temperature will at best be a mixture of brightness temperatures related to ice-free and ice-covered areas. The resulting brightness temperature is not necessarily representative for the examined grid cell, but is rather an average value for the adjacent footprints. If this is the case the resolution of the ice concentration field is thus further lowered according to the area covered by all nine footprints. It is also conceivable that solving the equation system leads to improper solutions for both the ocean and the land fraction.

\subsection{Correction for land spillover}

We found an approach to overcome the above-mentioned problem. The method described in this paper is similar to the approach of Bennartz (1999), although it was derived completely independent. The main difference is the field of application. Here we apply the method to improve sea ice parameters whereas the method of Bennartz (1999) was applied for the retrieval of atmospheric parameters.

We use eq. (3) to calculate the convolution of the antenna gain function with the land-water-distribution $\alpha$ for a given footprint in the coastal area. How the land fraction $\alpha$ is calculated for each measurement is described in Section 4.3. In a first step, the $\alpha$-values for all measurements within a pre-defined environment of the pixel to be corrected for the land impact are calculated. The selection of the environment is described in Section 4.4.

The $\alpha$-values for all measurements located within this environment are determined. For further analysis, only the footprints comprising mainly land surfaces are used. From these an average land brightness temperature for the respective environment is calculated. Two different weightings are performed to obtain the best land brightness temperature estimation for the footprint whose oceanic contribution to the measured overall brightness temperature shall be extracted.

First, the magnitude of the $\alpha$-values included in the averaging is used as a weighting factor to ensure that the footprints having the largest land fraction contribute most to the resulting representative land brightness temperature. On the other hand, the brightness temperature of land surfaces is locally quite variable. Thus, an additional weighting is performed taking into account the distance between the footprint location of the respective $\alpha$-value and the location for which a reasonable land brightness temperature shall be estimated. The weighting scheme used within this study is given in Section 4.4.

The obtained weighted mean for the land temperature $\left\langle T_{\text {land }}\right\rangle$ is then inserted into eq. (2) to calculate the ocean brightness temperature

$T_{\text {sea }}=\frac{T_{\mathrm{B}}-\alpha\left\langle T_{\text {land }}\right\rangle}{1-\alpha}$.

The resulting sea-only brightness temperature can then be inserted into the ice concentration algorithms.

\subsection{Land fraction calculation}

The definition of $\alpha$ given in eq. (3) contains the land mask $\mathrm{L}$ and the antenna gain function $\mathrm{G}$. The corresponding integral is taken over the elliptic footprint.

The considered footprint's exact location has to be known to insert the land mask data correctly when calculating $\alpha$. For this reason, the direction of the ellipse's major axis has to be determined. The major axis' declination angle can be separated into one component resulting from the satellite's flight track and one component resulting from the rotation of the scanning radiometer. A measurement's footprint declination relative to an arbitrary reference system is then given by

$\gamma=\varphi+\theta_{i}$

where $\varphi$ is the platform flight direction relative to the used reference system and $\theta_{i}$ is the scan angle relative to the flight direction. These two components can be derived directly from the SSM/I data. The approach used in this study is described in the next section.

In this study, the polar stereographic coordinate system is used as a reference system, but the described approach is valid for any ground-fixed Cartesian coordinate system. 
To simplify calculations, we introduce an auxiliary local reference system. The coordinates referring to this auxiliary reference system will be denoted by a prime in the following. This system has its point of origin at the centre point of the measurement for which the $\alpha$-value shall be calculated. Its $x^{\prime}$-axis orientation is defined by the orientation of the corresponding footprint ellipse's major axis relative to the original reference system.

Commonly the SSM/I antenna gain function is approximated by a 2-dimensional Gauss function. The actual antenna gain pattern is not fully characterized by a Gauss distribution, but has a complex three-dimensional structure including side lobes. However, it can be shown that a simple approximation using the Gauss function introduces an error in the order of $0.1 \%$ (Drusch et al., 1999). In the introduced auxiliary reference system the associated equation for the antenna gain function is

$G\left(x^{\prime}, y^{\prime}\right)=g_{0} \exp \left[-g_{1}\left(\frac{x^{\prime 2}}{a^{2}}+\frac{y^{\prime 2}}{b^{2}}\right)\right]=g_{0} \exp \left[-g_{1} r^{\prime 2}\right]$,

where $g_{0}, g_{1}$ are constants describing the Gauss distribution, $x^{\prime}$, $y^{\prime}$ are the distances in along-track and cross-track direction from the centre point of measurement and $a, b$ denote the semi-axes of the $-3 \mathrm{~dB}$ ellipse. The dimensionless elliptical radius $r^{\prime}$ is defined as

$r^{\prime}=\sqrt{\frac{x^{\prime 2}}{a^{2}}+\frac{y^{\prime 2}}{b^{2}}}$.

The $-3 \mathrm{~dB}$ radius is defined as the distance from the centre point where the antenna gain has decreased to half of its maximum value. Inserting this into eq. (6) yields $g_{1}=\ln 2$ (Drusch et al., 1999). Defining the total antenna gain to one gives $g_{0}=\frac{\ln 2}{\pi}$. Integration of the antenna gain function $\mathrm{G}$ to the radius $r_{-3 \mathrm{~dB}} / 2 r_{-3 \mathrm{~dB}} / 3 r_{-3 \mathrm{~dB}}$ results in $50 \% / 94 \% / 99.9 \%$ of the total gain (Drusch et al., 1999). In this study, the full resolution of $3 r_{-3 \mathrm{~dB}}$ is used. The threefold values of the $-3 \mathrm{~dB}$ footprints given in Table 1 are used for the integration interval in eq. (3) to calculate the $\alpha$-values related to the respective measurement's footprint.

In the local reference system, the points $\left(x^{\prime}, y^{\prime}\right)$ located within the threefold $-3 \mathrm{~dB}$ footprint ellipse are defined by

$\frac{x^{\prime 2}}{(3 a)^{2}}+\frac{y^{\prime 2}}{(3 b)^{2}} \leq 1$.

This equation thus describes the area over which the integral in eq. (3) has to be taken. The integral can be approximated by the following summation

$\alpha=\sum_{x^{\prime}=-3 a}^{3 a}\left(\sum_{y^{\prime}=-3 b f\left(x^{\prime}\right)}^{3 b f\left(x^{\prime}\right)} \bar{G}\left(x^{\prime}, y^{\prime}\right) L(x, y) \Delta y\right) \Delta x$,

where

$f\left(x^{\prime}\right)=\sqrt{1-\frac{x^{\prime 2}}{(3 a)^{2}}}$
Because the antenna gain function is defined relative to the auxiliary reference system $x^{\prime}$ and $y^{\prime}$ can be inserted straightforward in G. Whereas the land mask data set is given in polar stereographic coordinates and a transformation to the corresponding coordinates referring to the polarstereographic grid are required. The coordinates $\left(x^{\prime}, y^{\prime}\right)$ an arbitrary point has in the auxiliary reference system can be converted to its $x$ - and $y$-coordinates in the original reference system by

$\left(\begin{array}{l}x \\ y\end{array}\right)=\left(\begin{array}{cc}\cos \gamma & -\sin \gamma \\ \sin \gamma & \cos \gamma\end{array}\right)\left(\begin{array}{l}x^{\prime} \\ y^{\prime}\end{array}\right)+\left(\begin{array}{l}x_{c} \\ y_{c}\end{array}\right)$,

where $x_{c}, y_{c}$ are the coordinates of the centre point in the original reference system.

The sample rates $\Delta x$ and $\Delta y$ can be determined taking into account the footprint size, the land mask resolution and accuracy and the computational effort that can be provided.

\subsection{SSM/I scan geometry}

The total scan angle of the SSM/I radiometer is $\theta=102.4^{\circ}$. Within this angle the radiometer records 64 data points at the lower frequency channels and 128 data points at $85 \mathrm{GHz}$. This corresponds to an angle between subsequent samples of $\Delta \theta_{\text {low }}=$ $1.63^{\circ}$ for the $19 \mathrm{~V} / \mathrm{H}, 22 \mathrm{~V}$ and $37 \mathrm{~V} / \mathrm{H}$ channels and $\Delta \theta_{\text {high }}=$ $0.81^{\circ}$ for the $85 \mathrm{~V} / \mathrm{H}$ channels, respectively. For every measurement the sample number $i$ within the considered scan gives the declination of the corresponding measurement's ellipse major axis relative to the platform satellite flight direction

$\theta_{i}=\frac{1}{2} \theta-i \Delta \theta$

For the lower frequency channels $\Delta \theta=\Delta \theta_{\text {low }}$ has to be inserted, whereas for the $85 \mathrm{GHz}$ channels $\Delta \theta=\Delta \theta_{\text {high }}$. According to the number of samples per scan, $i$ ranges from 0 to 63 for the lower frequency channels and from 0 to 127 for the $85 \mathrm{GHz}$ channels, respectively.

To compute the platform flight direction $\varphi$ from the data, the satellite position projected to the Earth surface $\left(x_{\text {sat }}, y_{\text {sat }}\right)$ and its distance from the centre point of the recorded elliptic area on the Earth surface $r_{0}$ are considered. As the satellite moves forward during one scan sequence, $\left(x_{\text {sat }}, y_{\text {sat }}\right)$ is allocated to the satellite position at the beginning of the examined scan procedure. The coordinates refer to an arbitrary ground-fixed reference system. To illustrate the scan geometry and the approach to calculate the flight direction Fig. 3 shows a schematic sketch.

To simplify calculations an auxiliary coordinate system is introduced. This system has its origin in $\left(x_{\text {sat }}, y_{\text {sat }}\right)$ and its $x$-axis direction is coincident with the satellite navigation direction. The coordinates referring to this system are denoted by $x^{\prime}, y^{\prime}$. The centre point of the scan's $i$ th sample can be expressed by its $x$ - and $y$-component in the auxiliary coordinate system

$x_{i}^{\prime}=r_{0} \cos \theta_{i}+d_{i}$, 


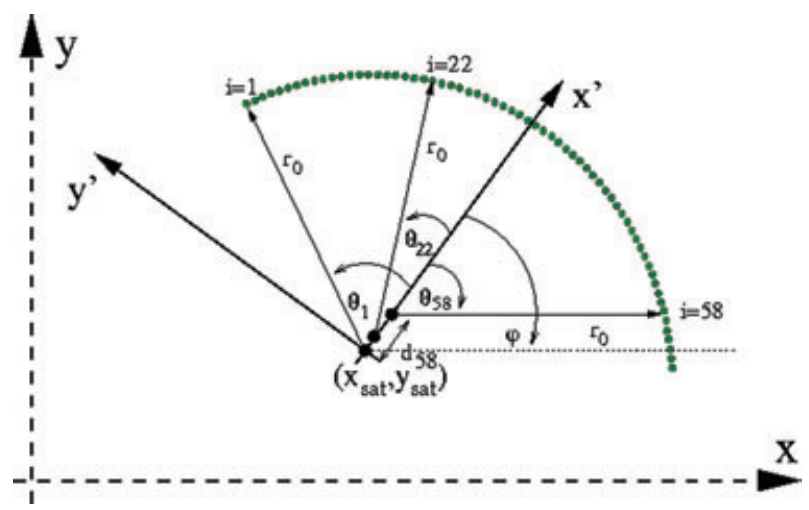

Fig. 3. Schematic scan geometry to illustrate how the satellite flight direction is calculated from the centre point positions given in the data. The green dots indicate the measurement's centre points, the red dots refer to the calculated satellite positions for different measurements $i$.

$y_{i}^{\prime}=r_{0} \sin \theta_{i}$,

where $d_{i}$ is the distance the satellite has covered from its position at the start of the scan sequence ( $\left.x_{\text {sat }}, y_{\text {sat }}\right)$.

The distance $d_{i}$ can be expressed by the satellite's flight velocity $v_{\text {sat }}$ that is assumed to be constant during one scan sequence and the time step between successive measurements $\Delta t$

$d_{i}=v_{\mathrm{sat}} i \Delta t$.

For the lower frequency channels $\Delta t$ is $8.44 \mathrm{~ms}$, for the $85 \mathrm{GHz}$ channels $\Delta t$ is $4.22 \mathrm{~ms}$.

The footprint centre positions expressed relative to the originally chosen arbitrary reference system are given by

$\left(\begin{array}{c}x_{i} \\ y_{i}\end{array}\right)=\left(\begin{array}{cc}\cos \varphi & -\sin \varphi \\ \sin \varphi & \cos \varphi\end{array}\right)\left(\begin{array}{c}x_{i}^{\prime} \\ y_{i}^{\prime}\end{array}\right)+\left(\begin{array}{c}x_{\mathrm{sat}} \\ y_{\mathrm{sat}}\end{array}\right)$.

Assuming that $N$ is the number of valid geolocations for the footprint centre positions an over determined equation system with $2 N$ known elements $x_{i_{1}}, y_{i_{1}}, \ldots, x_{i_{N}}, y_{i_{N}}$ and the five unknown parameters $x_{\text {sat }}, y_{\text {sat }}, v_{\text {sat }}, r_{0}$ and $\varphi$ can be set up. For this purpose the following arrays are defined

$G=\left(\begin{array}{ccccc}1 & 0 & \cos \theta_{i_{1}} & -\sin \theta_{i_{1}} & i_{1} \\ 0 & 1 & \sin \theta_{i_{1}} & \cos \theta_{i_{1}} & i_{1} \\ \cdot & \cdot & \cdot & \cdot & \cdot \\ \cdot & \cdot & \cdot & \cdot & \cdot \\ \cdot & \cdot & \cdot & \cdot & \cdot \\ 1 & 0 & \cos \theta_{i_{N}} & -\sin \theta_{i_{N}} & i_{N} \\ 0 & 1 & \sin \theta_{i_{N}} & \cos \theta_{i_{N}} & i_{N}\end{array}\right)$,

$\vec{m}=\left(\begin{array}{lllll}x_{\text {sat }} & y_{\text {sat }} \quad r_{0} \cos \varphi & r_{0} \sin \varphi & v_{\text {sat }} \Delta t \cos \varphi\end{array}\right)^{\mathrm{T}}$,

$\vec{d}=\left(\begin{array}{lllllll}x_{i_{1}} & y_{i_{1}} & \cdot & \cdot & x_{i_{N}} & \left.y_{i_{N}} \cdot\right)^{T}\end{array}\right.$
The equation system $G \cdot \vec{m}=\vec{d}$ has to be solved for $\vec{m}$. $G$ can be factorized using singular value decomposition. The $(2 N \times$ 5)-matrix $G$ of rank $p$ is then expressed by

$G=U_{p} S_{p} V_{p}^{\mathrm{T}}$,

where $U_{p}$ is a $(2 N \times p)$-matrix, $S_{p}$ is a $(\mathrm{p} \times \mathrm{p})$-diagonal matrix and $V_{p}^{\mathrm{T}}$ is a $(p \times 5)$-matrix. The least squares solution for $\vec{m}$ is the solution minimizing $\|G \vec{m}-\vec{d}\|$ and is obtained from

$\vec{m}=V_{p} S_{p}^{-1} U_{p}^{\mathrm{T}} \cdot \vec{d}$

The satellite flight direction $\varphi$ is then extracted from the least squares solution for $\vec{m}$.

\subsection{Performance considerations}

The separation method can be performed using different realizations of the basic idea described in the previous sections. There are mainly five aspects that can be varied. One of these is the selection of the search area from which footprints with high $\alpha$-values are taken to determine an adequate land brightness temperature. In addition, a threshold for the $\alpha$-values to be considered in the averaging is required. Furthermore, the weighting procedures related to the magnitude of $\alpha$ and the associated footprint's location have to be considered. Finally, the sample rate for the $\alpha$-calculation can be modulated.

Each of these decisions deals with the problem of finding the best compromise between two sometimes contradictory requests. On the one hand, it is reasonable to include only footprints with very high land fraction in the averaging process. On the other hand, the brightness temperature variation over land requires to confine the included pixels to the ones located as close as possible to the pixel to be corrected for land spillover. All realizations quoted in the following are chosen rather arbitrarily. However, they seem to work reasonably. Moreover, application of the separation method to SSM/I data using different adequate variations of these realizations showed to have only minor effect on the results. This is especially true as the results are compared only qualitatively within this study.

The separation method searches for pixels representing land surfaces within the search area of any measurement to be corrected for land spillover. Several definitions for this search area are conceivable. Within the scope of this study we used elliptic search areas. The eccentricity and the declination of these ellipses correspond to the ones of the channels' footprints. This definition does not only take into account the centre pixels' positions but also the shape of the footprint and thus the area contributing to each measurement.

The required size of the search area depends on the local conditions, the footprint size and the threshold value for $\alpha$ 's used for averaging. The search area has to be chosen sufficiently large to ensure that within this area footprints containing mainly land surfaces are found. We chose a threshold value of $\alpha_{\min }=0.95$ 
for pixels considered as representative for land surfaces. For applications in the Sea of Bothnia we thus found the optimal size of the search area in terms of multiples of the channel's corresponding footprint size. For the 19 and $22 \mathrm{GHz}$ channels the ellipses had to be four times the corresponding $-3 \mathrm{~dB}$ footprint ellipses. For the 37 and $85 \mathrm{GHz}$ channels, the factor was 5 and 10 , respectively. For these values we found pixels representing land within the search area of all coastal measurements to be corrected for land impact. The given multiples of the footprint ellipses represent the areas where the pixels' centre points are located. If the $-3 \mathrm{~dB}$ footprint size is added to the resulting search areas they correspond to roughly $310 \mathrm{~km} \times 190 \mathrm{~km}, 270 \mathrm{~km} \times 180 \mathrm{~km}$, $200 \mathrm{~km} \times 150 \mathrm{~km}$ and $160 \mathrm{~km} \times 140 \mathrm{~km}$ for the $19,22,37$ and $85 \mathrm{GHz}$ channels, respectively.

All $\alpha$-values included in the averaging process were assigned two weighting factors. One factor according to the magnitude of $\alpha$ and one according to the pixel location. The resulting weighting factor is then the product of these factors. The weighting according to the magnitude of $\alpha$ was chosen to be normally distributed between the maximum value of 1 and the minimum value $\alpha_{\min }=0.95$. We chose the distribution width so that the weighting factor decreases to half of its value for every 0.01 step of $\alpha$. Accordingly, pixels with $\alpha=0.99$ are weighted only half as much as pixels with $\alpha=1$, for example. The weighting related to the considered pixel's location was as well chosen to be normally distributed between the position of the pixel to be corrected for land spillover and the position of the pixels located on the search area's margin. Therefore, we divided the elliptic search area into five concentric elliptic regions with equidistantly increasing semi-axes. We chose the distribution width so that the weighting factor decreases to half of its value when going from one of these elliptical regions to the neighbouring one. Pixels located on the margin of the innermost ellipse are thus weighted twice as much as pixels located on the margin of the neighbouring ellipse, for example.

We brought the land mask data to a grid spacing of $500 \mathrm{~m} \times$ $500 \mathrm{~m}$. The summation in eq. (9) was as well performed with a sampling rate of $500 \mathrm{~m}$ in $x$ - and $y$-direction, respectively.

\section{Results}

We have applied our method to calculate the ice concentration in the Sea of Bothnia located in the northern Baltic Sea. To compare our results to the actual ice concentration field in this region, we selected some example days in March and April 2009. The choice of these days was restricted by the simultaneous availability of suitable MODIS and ASAR images.

As a first step we adjusted the tie points used in the ASI algorithm to Baltic conditions (Section 5.1). In Section 5.2, the adaption of the weather filter thresholds to Baltic conditions is described. Thereafter the ice concentrations calculated with both the NASA Team and the ASI ice algorithm are shown for the uncorrected SSM/I data and the separated SSM/I data, respectively (Section 5.3). These are then compared to MODIS and ASAR images.

\subsection{ASI algorithm tie point adaption}

The ASI algorithm requires characteristic $85 \mathrm{GHz}$ polarization differences for open water and complete ice coverage, respectively. One possible method to obtain these tie points for the Baltic Sea is to choose two areas of examination. One of them is supposed to be completely ice covered during the period of examination and the other is supposed to be ice-free. We selected one area located in the northern Bay of Bothnia and used Finnish and Swedish ice charts to determine days in 2008 and 2009 , when the ice concentration within this area was $100 \%$. All together 12 days in March 2008 and 30 days in February/March 2009 matched this criterion. The second area is located further south and was ice-free at the examined days.

For the resulting tie point of open water, we obtained an average value of $45.0 \mathrm{~K}$ with a standard deviation of $9.2 \mathrm{~K}$. Hence the tie point found for open water is very close to the one used for Arctic conditions. Spreen et al. (2008) give $46 \mathrm{~K}$ as an optimal tie point.

For the ice-covered region averaging leads to a mean value of $21.7 \mathrm{~K}$ with a standard deviation of $15.0 \mathrm{~K}$. Shokr et al. (2009) investigated the behaviour of microwave radiation emitted by thin artificial sea ice grown in an outdoor tank. They found that the $85 \mathrm{GHz}$ polarization difference increases, for example when the bare-ice surface starts to melt or immediately after snow starts to fall. The ice charts for the examined days indicate that the sea ice distribution during the examined days was very variable with alternating periods of melting and freezing sea ice. Thus, according to the results obtained in Shokr et al. (2009) the mean value of $21.7 \mathrm{~K}$ is not necessarily the best value representing $100 \%$ ice concentration, but rather an upper boundary for the tie point.

To find a more representative ice tie point, we used SSM/I brightness temperatures from several days to generate ASI ice concentration maps. The ice tie point was varied and the resulting ice concentration fields were compared to high-resolution satellite images and ice charts. We obtained the best results for an ice tie point value near $16 \mathrm{~K}$. This value appears reasonable as it is between the value Spreen et al. (2008) found to be representative for Arctic conditions (7.4 K) and the upper boundary obtained from averaging all measured polarization differences $(21.7 \mathrm{~K})$.

\subsection{Adjusting the weather filter thresholds}

To obtain optimal weather filter thresholds for Baltic Sea applications, we chose one area that was always ice-free during the examined time period and one area containing at least partly ice during that period. The chosen areas are roughly the same as were used for the tie point determination. The period of 
examination was again selected by studying Swedish and Finnish ice charts for the winter seasons 2008 and 2009. The SSM/I swath data for the period January 30 to May 1, 2008 and January 15 to May 20, 2009 were then included in the analysis.

The swath data was used to calculate the gradient ratios GR(37V, 19V) and GR(22V, 19V). Simultaneously, the mean polarization difference $\mathrm{P}(85)$ was determined. As the weather filters are used to reduce ice concentration overestimation, only cases where $\mathrm{P}(85)$ was below the open water tie point of $45 \mathrm{~K}$

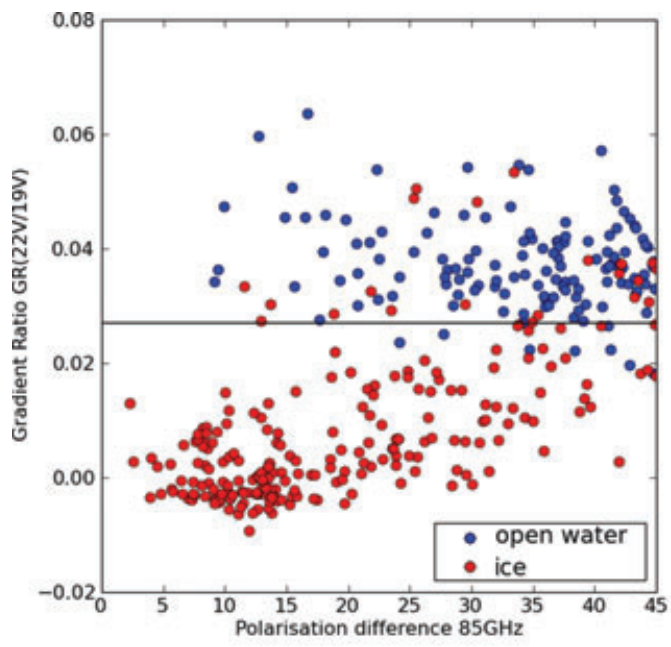

(a) $\mathrm{GR}(22 \mathrm{~V}, 19 \mathrm{~V})$, freezing conditions

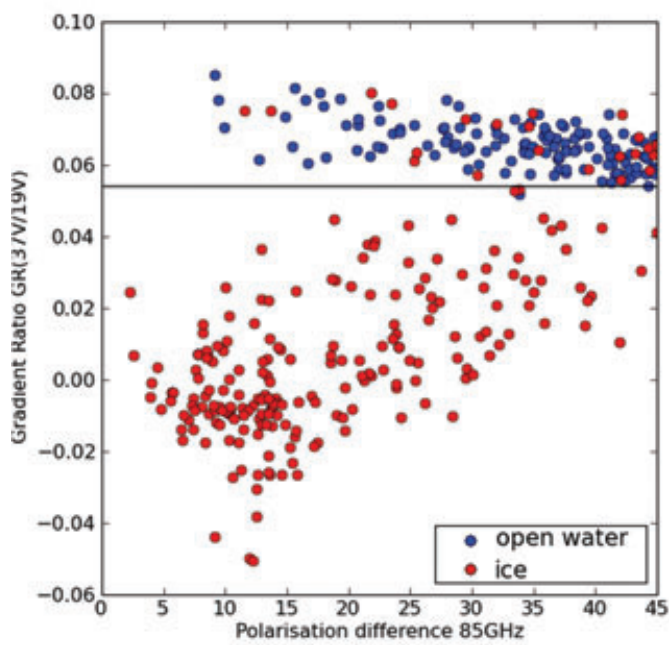

(c) GR(37V,19V), freezing conditions were analysed. In the strict sense this approach is restricted to the ASI algorithm usage. However, we assume that constraining the gradient ratio analysis to low polarization differences does not introduce serious limitations concerning the weather filter usage in connection with the NASA Team algorithm.

The optimal threshold was defined to be the value associated with the lowest number of false assignments. If the minimum error rate could not be determined uniquely, the average value of the gradient ratios associated with the minimum number of false assignments was selected.

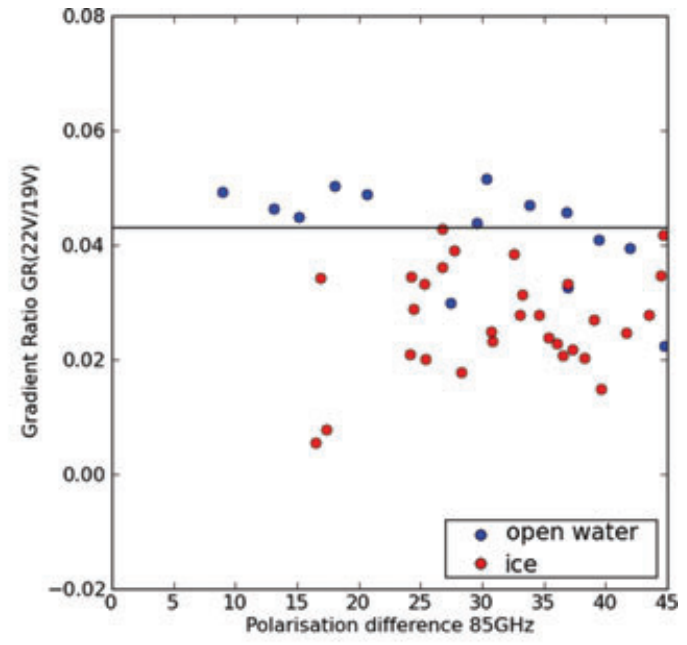

(b) GR(22V,19V), melting conditions

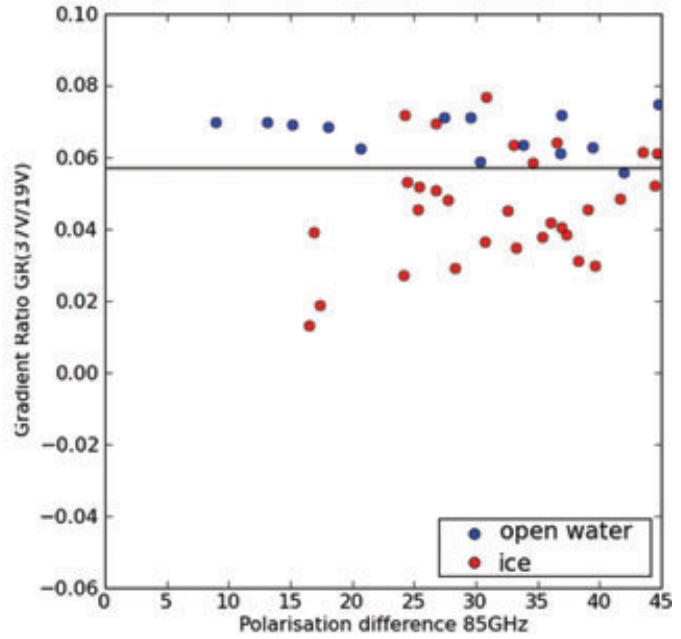

(d) GR(37V,19V), melting conditions

Fig. 4. Gradient ratios $\mathrm{GR}(22 \mathrm{~V}, 19 \mathrm{~V})$ and $\mathrm{GR}(37 \mathrm{~V}, 19 \mathrm{~V})$ as functions of the $85 \mathrm{GHz}$ polarization difference $\mathrm{P}(85)$. The subfigures show measured GR(22V, 19V)-values for freezing (a) and melting conditions (b) and the measured GR(37V, 19V)-values for freezing (c) and melting conditions (d). The blue dots refer to data points obtained in the open water area, red dots refer to the at least partly ice-covered examination area. The black line indicates the chosen optimal threshold value. For the weather filter based on GR $(22 \mathrm{~V}, 19 \mathrm{~V})$ the analysis yields an optimal threshold value of 0.027 for freezing conditions and 0.043 for melting conditions. For the GR(37V, 19V) weather filter an optimal threshold value of 0.054 for freezing conditions and 0.057 for melting conditions was obtained. 
Table 2. Weather filter thresholds and rate of false assignments associated with the chosen threshold values

\begin{tabular}{lccccc}
\hline & \multicolumn{2}{c}{ Melting } & & \multicolumn{2}{c}{ Freezing } \\
\cline { 2 - 3 } \cline { 5 - 6 } & Threshold & Error rate & & Threshold & Error rate \\
\hline GR(22V/19V) & 0.043 & 5 of 44 (11.4\%) & & 0.027 & 30 of 358 (8.4\%) \\
GR(37V/19V) & 0.059 & $8-9$ of 44 (18.2-20.5\%) & & 0.053 & 24 of 358 (6.7\%) \\
\hline
\end{tabular}

When the ice concentration algorithms were applied to SSM/I data using the new weather filter thresholds some difficulties arose in areas where melting conditions were found. The weather filters caused an underestimation of ice occurrence under these circumstances. Consequently we analysed the time period when melting occurred in the ice covered area separately. In the last days of the respective winter season the ice cover was obviously retreating towards ice-free conditions. We thus chose the time periods 20 April to 1 May 2008 and 1 May to 20 May 2009 to represent melting conditions. However, as the ice coverage in the Baltic Sea is quite variable, melting and freezing occur certainly as well in the earlier stage of the winter season, but possibly less pronounced.

Fig. 4 shows the results. The optimal threshold values and the respective occurrences of false assignments are given in Table 2. Under melting conditions the optimal threshold values were found to be higher than for non-melting conditions. This is especially true for the $\mathrm{GR}(22 \mathrm{~V}, 19 \mathrm{~V})$ values.

\subsection{Ice concentration fields}

To test the developed separation method it was applied to SSM/I data as described in Section 4. The area under examination lies within a rectangular box in the Sea of Bothnia and is depicted in Fig. 5.

As a first step we identify the swath tracks containing data located in the area of investigation and convert the data point positions into polar-stereographic coordinates. The swath track with the maximum number of valid brightness temperature measurements for any examined day is then selected.

For each channel, the area in which land surfaces have an impact on the measured brightness temperature is determined. This includes the coastline and any islands in the area of investigation and the surrounding area within a distance of twice the main-axis of the considered footprint, corresponding to four times the main-axis of the $-3 \mathrm{~dB}$ ellipse given in Table 1 . This land affected area is computed automatically using a Sobel filter to detect edges in the land mask and broadening the obtained line to satisfy the requirements. In the Bothnian Bay, this proceeding is not necessary for the lower frequency channels with their large footprint sizes but in case of the $85 \mathrm{GHz}$ channels and their considerably smaller footprints this pre-selection of affected re-

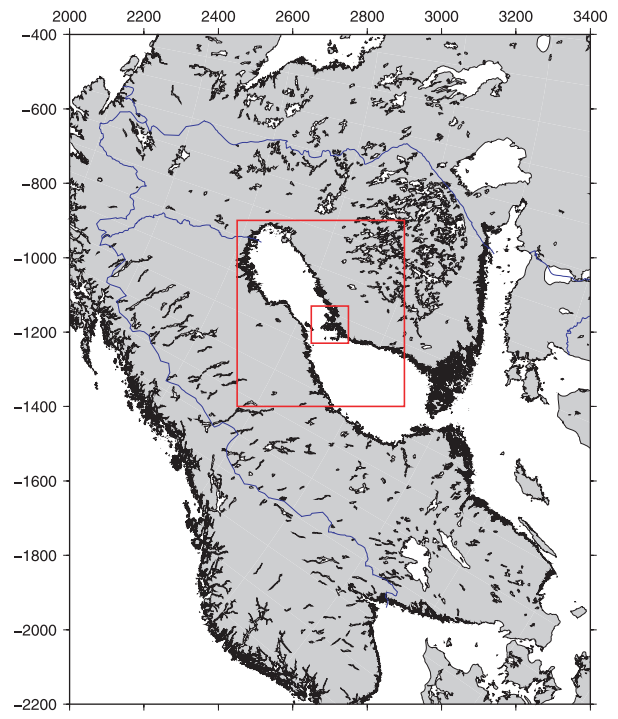

Fig. 5. Examination area in the Baltic Sea. The larger rectangle indicates the area in the Sea of Bothnia for which the separation method was tested. The smaller rectangle depicts the region shown in Fig. 11.

gions can be useful to avoid unnecessary calculations. This can also be true for the remaining channels when applied to retrieve ice concentrations in the Arctic.

The separation method described in the previous section is then performed for all pixels with $0.05 \leq \alpha \leq 0.95$.

To compare our results to high-resolution satellite images, we selected some example days in the winter season 2009 for which ASAR images were available and the weather conditions were favourable concerning the MODIS records. The resulting ice concentration fields for two example days are shown in the Figs 6 and 8 . The depicted ice concentration fields are interpolated to a $2.5 \mathrm{~km} \times 2.5 \mathrm{~km}$ grid. However, it should be kept in mind that decreasing the pixel size for depiction purposes does not provide any additional information.

On 27 March 2009 (Fig. 7), the ASAR and MODIS images show that the Bay of Bothnia was almost completely covered by ice except for a tongue of open water extending from the southwestern coast northwards. At the eastern coast the ice reaches further south in the Gulf of Bothnia. The Finnish archipelago off 


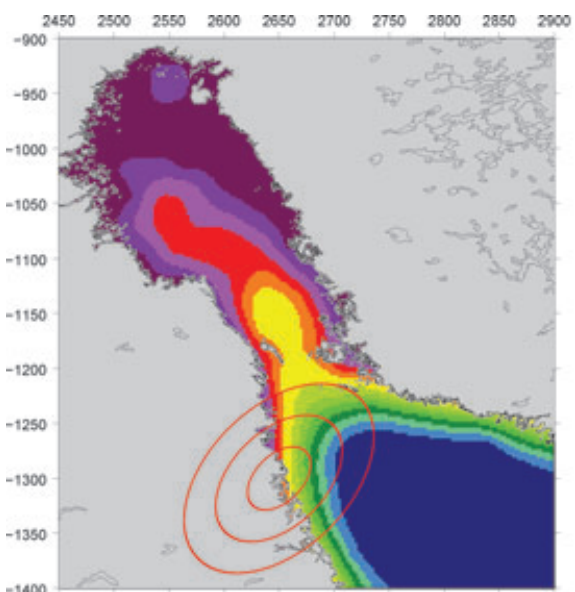

(a) NASA Team original

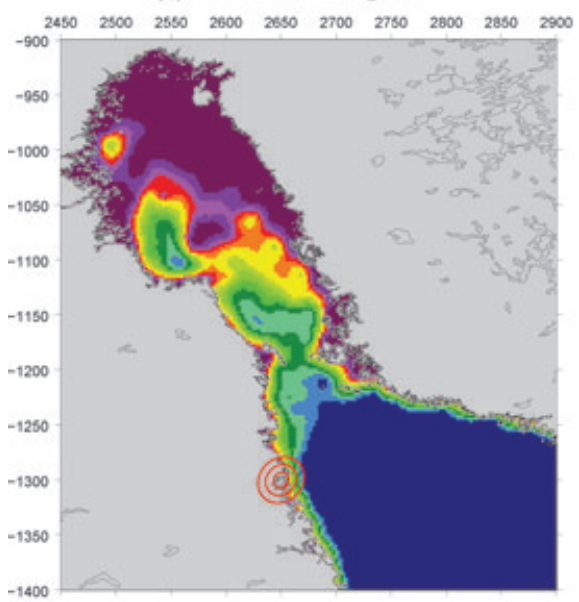

(c) ASI original

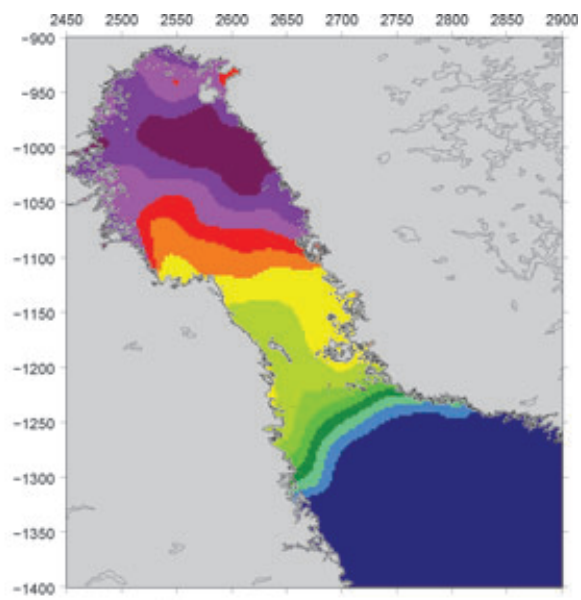

(b) NASA Team corrected

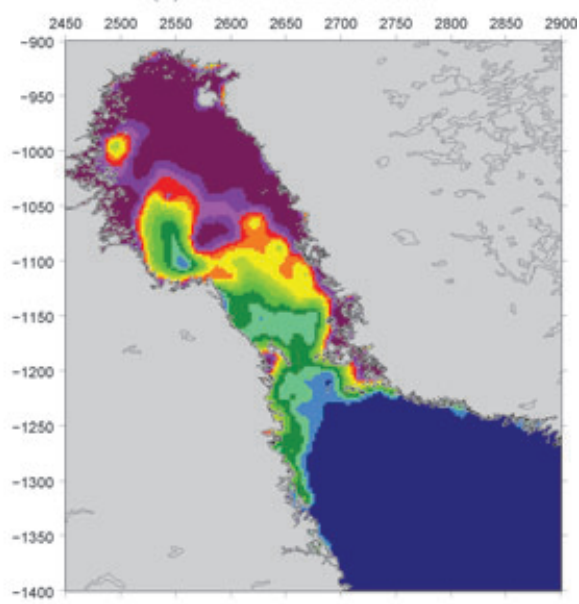

(d) ASI corrected

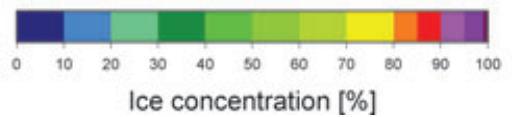

Fig. 6. Ice concentrations retrieved with NASA Team (top) and ASI algorithm (bottom) for 27 March 2009. On the left-hand side are the ice concentrations obtained from uncorrected SSM/I brightness temperatures. On the right-hand side are the corresponding results after correction for land spillover. The $-3 \mathrm{~dB}$ footprint and its doubled and threefold areas are indicated for the $19 \mathrm{GHz}$ (top) and $85 \mathrm{GHz}$ (bottom) channels.

Vaasa located approximately at the same geographical latitude as the Swedish island Holmön is surrounded by fast ice. In addition, the Holmön island that is depicted in Fig. 2 is connected to the mainland by fast ice.

For the ice concentration retrieval on 27 March, the weather filters for freezing conditions are used. Applying the NASA Team algorithm to the uncorrected SSM/I data roughly reveals the open water tongue, but produces a rather broad band of ice occurrence along the entire coastline. The ice concentration near the south-western coast of the Bothnian Bay is slightly underestimated as is the fast ice extent in the vicinity of Holmön and the archipelago off Vaasa.

When we insert the corrected SSM/I brightness temperatures in the NASA Team algorithm, the coastal ice band resulting from the land impact disappears. The ice occurrence near Holmön and the archipelago off Vaasa are still indicated, but the ice concentration is underestimated. In the northernmost part of the Bothnian Bay, the separation method causes a slight decrease of ice concentration.

Due to the higher resolution of the $85 \mathrm{GHz}$ channels, the effect of applying the separation method to the SSM/I data is less obvious for the ASI algorithm ice concentration fields. Using the ASI algorithm with the uncorrected SSM/I brightness temperatures again leads to a continuous band of ice occurrence along the coast, whereas the land impact is narrower compared to the NASA Team algorithm. Using only the ocean contribution for coastal data points removes this erroneous detection of ice, while ice actually present in coastal areas is maintained, as for example the fast ice surrounding Holmön and the archipelago off Vaasa. 


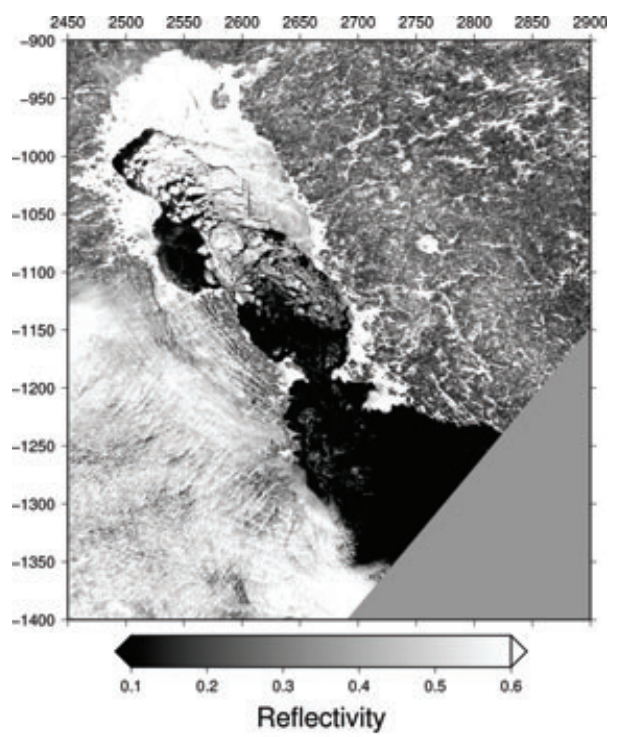

(a) MODIS

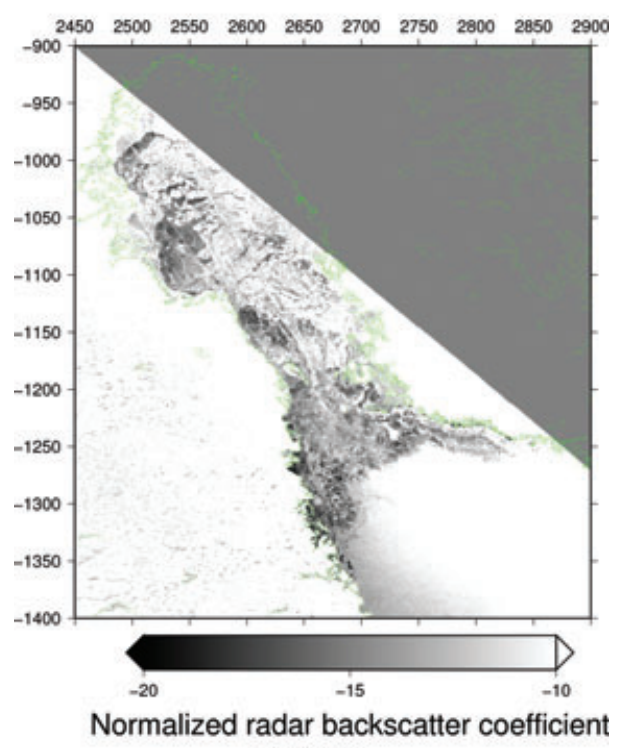

(b) ASAR

Fig. 7. MODIS (top) and ASAR (bottom) images taken on 27 March 2009.

In the MODIS image of this day it is difficult to distinguish between dark sea ice areas and open water areas. The MODIS image seems to indicate a larger open water area than the ASAR image. The ice edge retrieved with the separated SSM/I data resembles the ice edge seen in the ASAR image. This example day indicates how difficult it is to retrieve ice concentrations from MODIS and ASAR images.

For the ice concentration retrieval on 23 April 2009, the weather filter for melting conditions was used, as the ice was retreating gradually, especially in the southern part of the examined area. Figure 8 shows the strong land influence on ice concentration retrieval in actually ice-free parts of bays being embedded by land from both sides. This is especially true for algorithms using the lower SSM/I channels. When the uncorrected SSM/I data is used, the NASA Team algorithm for instance reveals no open water where the Sea of Bothnia is narrowest. Although this area is actually completely ice-free. Applying the separation technique leads to ice concentration fields rather similar to the ones obtained with MODIS and ASAR (see Fig. 9) for both algorithms. Due to the higher resolution of the ASI algorithm, the open water in the south-western Bothnian Bay is depicted even more detailed. The erroneous ice concentration values along the coast vanish when the separation method is applied. Only one small area near the south-western coast of the Sea of Bothnia is assigned an ice concentration of approximately $40 \%$ that cannot be found in the MODIS image. In this area, the ASAR image shows a large pattern of high backscatter values that is hard to interpret. But as the ice edge is far north and the surroundings show merely open water areas, this seems to be an erroneous indication of sea ice. It is remarkable that the small band of ice along the western coast of the Bothnian Bay is maintained. As is the fast ice around the archipelago off Vaasa. Although the fast ice concentration is slightly underestimated by both algorithms.

For some cases where the ice concentrations appeared to be underestimated it should be considered whether these low concentrations may also be allocated to the coarse resolution of the involved channels. If the large footprint size of the lower resolution channels is imagined to be overlied to such a pixel the given ice concentration values might be reasonable.

Figure 10 shows only the corrected coastal ice concentration values for 23 April 2009. Within this context the coastal pixels are defined as the pixels with $0.05 \leq \alpha \leq 0.95$, that is all pixels included in the process of correction for land spillover. In these figures the ice concentration values are shown without applying any interpolation. Moreover, no land mask is overlaid to the obtained ice concentration values. The size of the square boxes corresponds to the size of the involved SSM/I channels' sample spacing. It should be noted that this is only the grid spacing of the measurements and not the resolution which is given by the footprint size. The square boxes are declined according to the orientation of the corresponding footprints. It exposes the grid cell distribution as measured with SSM/I instead of interpolating the data points to the rectangular coordinate system specified by the polar stereographic projection.

In this depiction, the corrected brightness temperatures are not overlayed by a land mask and the values retrieved for pixels whose centre points are located over land are visible. Because only the pixels corresponding to $\alpha$-values greater than 0.95 are included in the correction processing, all pixels shown in the figures have footprints with an ocean contribution of at least $5 \%$. However, it should be noted that the separation method may introduce quite large errors for high $\alpha$-values. Thus pixels not located in the direct vicinity of the coast should be treated carefully. The area where the obtained brightness temperatures can be included in the interpretation of the images depends on the 


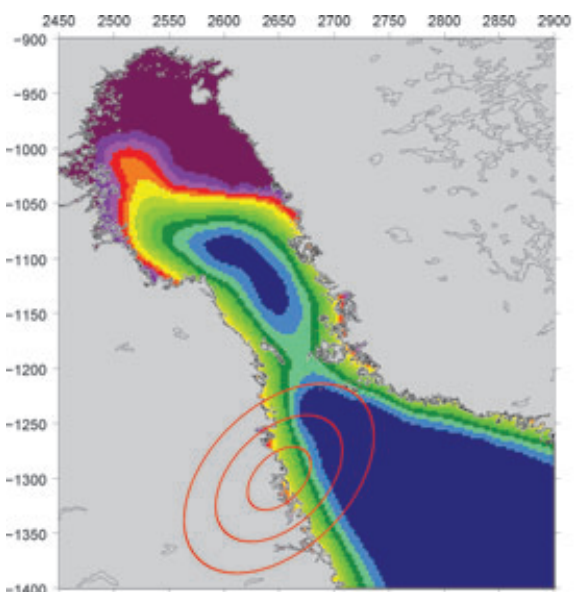

(a) NASA Team original

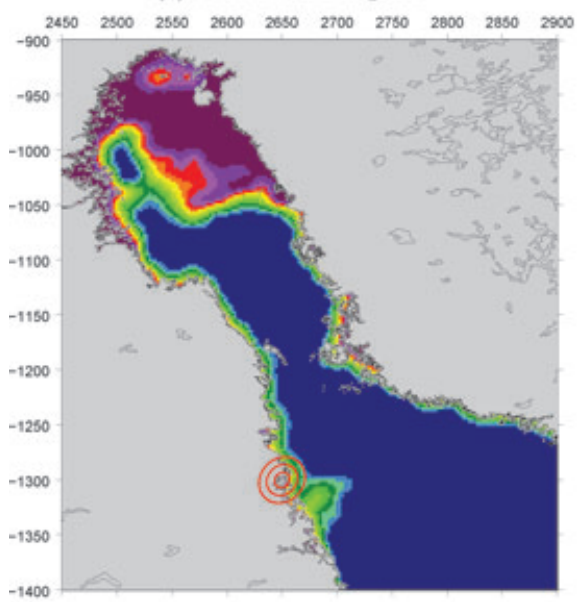

(c) ASI original

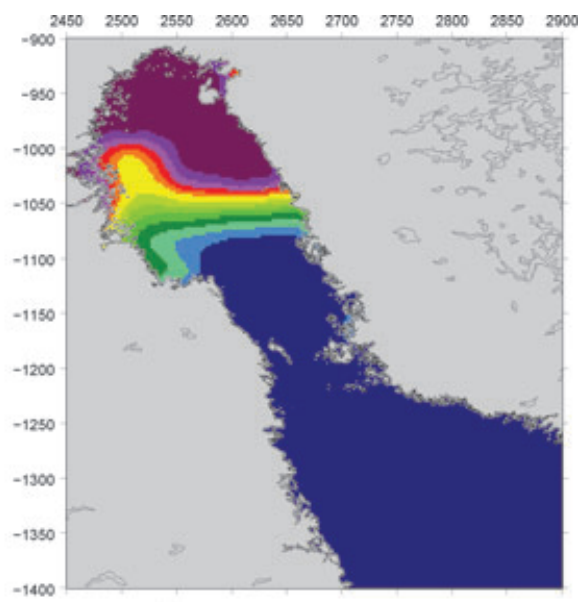

(b) NASA Team corrected

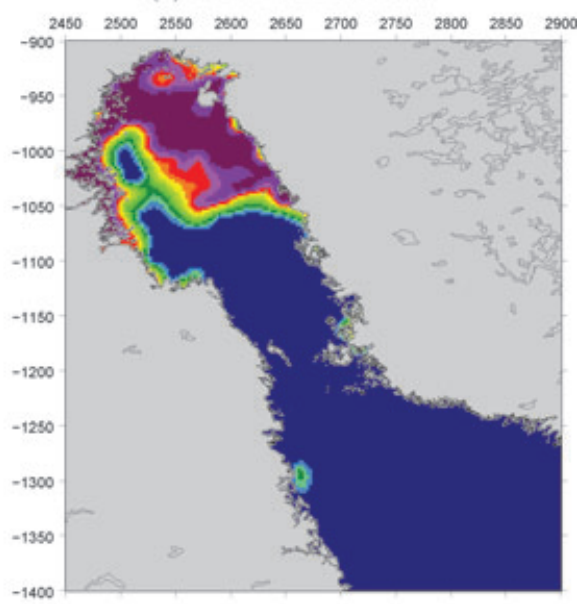

(d) ASI corrected

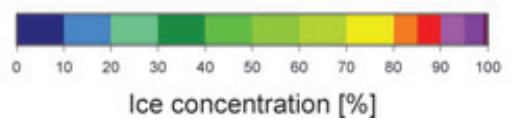

Fig. 8. 23 April 2009. For figure description, see caption of Fig. 6.

footprint size. It is of course smaller for the $85 \mathrm{GHz}$ pixels than for the lower resolution pixels. Considering the sample spacing and the footprint sizes, as a first approximation all pixels whose margins do not cross the shoreline can be ignored.

The remaining pixels located over land can be interpreted as follows. Each pixel represents the brightness temperature distribution within the corresponding footprint. In the land spillover correction process the land contribution is supposed to be subtracted. The remainder is then assumed to represent the ocean contribution for this footprint. Due to the much larger footprint size as compared to the pixel size, this signature may be related to an area lying outside the pixel in the adjacent sea.

For some areas where coastal ice concentration retrieved with the corrected SSM/I data was underestimated compared to the MODIS and ASAR images some interesting features are visible in the figures not overlayed with the landmask. The red arrow in Fig. 10b indicates an ice concentration of $100 \%$ retrieved with the ASI algorithm. For the same region the NASA Team algorithm as well indicates ice (Fig. 10a). In Fig. 8 these pixels were hidden by the landmask. Figure 11 shows an enlarged ASAR image of the Finnish archipelago off Vaasa as depicted in Fig. 5. It clearly indicates ice in the considered region. Possible explanations are discussed in the next section.

\section{Discussion}

The correction of SSM/I brightness temperatures for land spillover in the Baltic Sea was carried out successfully. Implementation of the separation method to the NASA Team and the ASI sea ice concentration algorithms thus appears to be very promising. Comparison of the obtained ice concentrations with MODIS and ASAR images generally revealed a good agreement. 


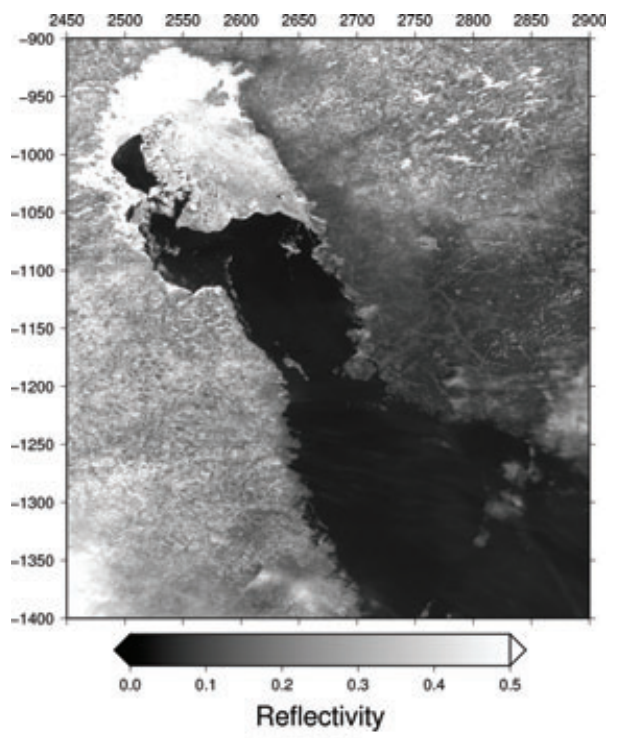

(a) MODIS

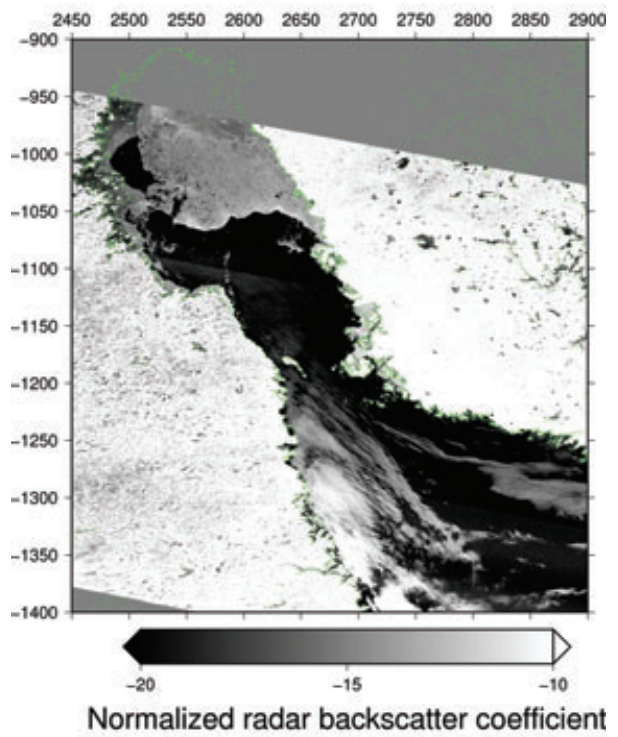

(b) ASAR

Fig. 9. MODIS (top) and ASAR (bottom) images taken on 23 April 2009.

Observed deviations from the true ice situation can emerge from several sources that will be discussed in the following.

A feature that can greatly affect the accuracy of the separation method is the SSM/I instrument's geolocation error. A shift of a given pixel position from the actually measured area's centre point position leads not only to a brightness temperature value not representative for the considered area. Due to the required knowledge of the land-water-distribution within the considered footprint, for calculating the $\alpha$-values any geolocation error introduces an additional error. Geolocation errors may have caused the effects depicted by red arrows in Fig. 10. Here ice concentrations seemed to be underestimated after the correction for land impact, but appropriate ice concentration values were found to be hidden under the overlaid landmask.

The SSM/I geolocation error has been estimated for the different platforms (Hollinger, 1990; CAL/VAL 1998; Bennartz, 1999). For the F8, F10 and F11 platforms the geolocation error ranges between \pm 5 and $\pm 10 \mathrm{~km}$. Compared to the footprint size a geolocation error in this order of magnitude introduces a considerable source of error, particularly for the $85 \mathrm{GHz}$ channels. The geolocation information is supposed to be retrieved more accurately in the future. The separation method is clearly going to benefit from an improved accuracy of geolocation information. This is particularly true for the processing of higher resolved microwave data, as seen in the $85 \mathrm{GHz}$ channels of SSM/I or in the high-frequency channels of AMSR-E.

The underlying landmask was brought to a grid spacing of $500 \mathrm{~m}$, although for the Baltic Sea area more highly resolved landmasks are available. The $\alpha$-values were calculated by adding up the value obtained from multiplying the antenna gain function with the characteristic value associated with the land-waterdistribution every $500 \mathrm{~m}$ in scan- and flight-direction, respectively. For the present-day accuracy of geolocation information, the high landmask resolution and sampling rate are probably not necessary. This is confirmed by the fact that the results obtained for a sampling interval of $1 \mathrm{~km}$ were quite similar to the ones obtained with a sampling interval of $500 \mathrm{~m}$. However, the higher sampling rate was used in this study because the computational effort for an area comparable to the examined Baltic Sea region is in acceptable limits. If the method is applied to more prolonged areas at least for the lower resolution channels a lower sampling rate should be considered. If the geolocation information becomes more accurate in the future the separation method is supposed to improve with decreasing sampling intervals.

The calculations of the $\alpha$-values were performed using the threefold $-3 \mathrm{~dB}$ footprints. However, when only the doubled $-3 \mathrm{~dB}$ footprints were used, the obtained results did not differ fundamentally.

Together with the above-mentioned considerations about the impact of the landmask resolution and sampling rate, the latter issue is an indication for the significance of the mentioned error sources related to the land fraction calculation which is a fundamental part of the separation method. A possible interpretation yields the predominance of the geolocation errors compared to other simplifications and limitations.

Another source of error is the assumption of constant land brightness temperatures to extract the ocean contribution. If the land brightness temperature within the examined area is almost constant, the resulting average value can be assumed to be representative for the land fraction of the examined measurement. However, if the land brightness temperature is very variable this assumption will introduce a larger error. The difference between ocean and land brightness temperatures is quite high. Thus, our separation method provides an improvement for coastal ice concentration retrieval despite the error introduced by the 


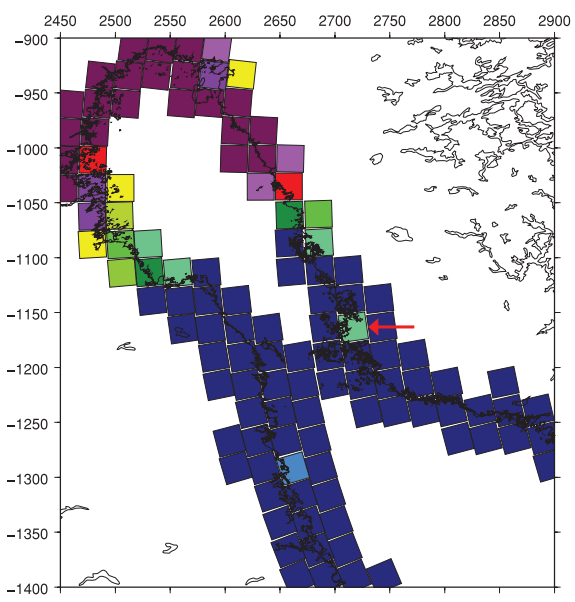

(a) NASA Team

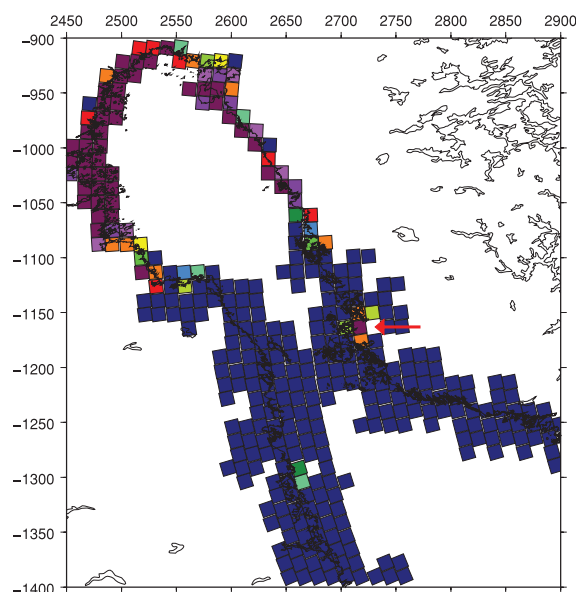

(b) ASI

Fig. 10. Corrected coastal ice concentrations for 23 April for the NASA Team (left-hand side) and the ASI (right-hand side) algorithm. The size of the square boxes corresponds to the sample spacing of $25 \mathrm{~km} \times 25 \mathrm{~km}$ and $12.5 \mathrm{~km} \times 12.5 \mathrm{~km}$ for the lower frequency channels and the $85 \mathrm{GHz}$ channels, respectively. They are declined according to the declination of the footprint major axis.

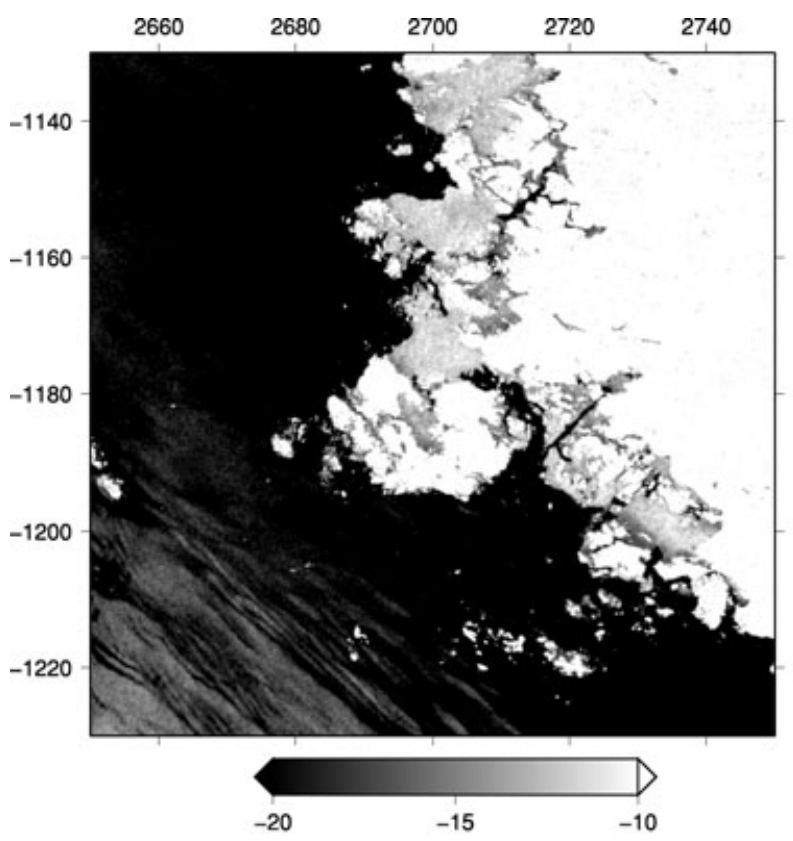

Normalized radar backscatter coefficient

Fig. 11. ASAR image of the archipelago off Vaasa. The illustrated region is depicted in Fig. 5.

variability of the land brightness temperature. Because we used single swath data, the temporal variability of the land brightness temperature does not influence the obtained results. To reduce the errors introduced by the spatial variability of land signatures, we minimized the area from which land brightness temperatures were taken to determine an average value.

The analysed ice concentration fields were picked by the simultaneous availability of ASAR records and suitable MODIS images. Because the used MODIS band is in the visible light spectrum, the sea surface is observable only if the overlying atmosphere is cloud-free. Thus, the exemplary days analysed in this study are probably ice concentration retrievals conducted under rather favourable conditions.

However, atmospheric phenomena apart from clouds can cause erroneous ice concentrations. These include water vapour content and the liquid water path, for example. The atmospheric conditions encountered at the time the ice concentration is retrieved should be considered for interpretation of the results. By this means it could be distinguished between false ice assignments allocated to the present atmospheric conditions and to limitations of the separation method, respectively.

The adjustment of the weather filter thresholds and the tie points to Baltic Sea conditions showed that the considered values were rather variable. The used approach is only a first approximation to a determination of optimal values. However, the analysis demonstrated the difficulties arising when pre-determined values are used for all ice and weather situations.

Considering the weather filters for freezing and melting conditions separately is perhaps a promising approach. The determination of weather filter thresholds for melting conditions could be improved by including more data. However, this approach will lead to a more complex treatment, especially if melting and freezing take place simultaneously in different areas of the examined region.

The resulting ice concentration fields will be more consistent and realistic if not only single swath data are used but the average of several overflights. The effect of single erroneous measurements would be reduced in the process of averaging. Another promising approach could be to search for measurements whose centre points are located close to each other. But the satellite flight directions and thus the footprint declinations should be 
different, in the best case declined in opposite directions. In that case the mean value of the two (or more) resulting ocean brightness temperatures actually represents an average value for the area surrounding the assumed approximately same centre point whereas the centre region contained in both footprints is double weighted. This approach would of course be more effective for footprints having a more elliptic shape compared to nearly circular footprints. For the SSM/I channels the effect would thus decrease with increasing frequency as the excentricity of the footprint ellipses decreases.

Because neither the definition of the search size nor the different weightings or the selection of $\alpha_{\text {min }}$ seemed to have essential impact on the separation method performance, the method seems to be rather robust. Nevertheless, the influence of these modulations should be investigated further to allow for more precise conclusions.

The ice concentration algorithms used in this study are merely exemplary applications for the presented separation technique. The method can as well be used for other sea ice retrieval models. Moreover, the developed approach can potentially improve other parameter retrievals if more than two different surface types are involved.

Due to the moderate computational effort required for land spillover correction using the presented separation method it is suitable for implementations in the processing chain of numerical weather prediction models, for example.

\section{Conclusions}

Our aim was to remove the systematic error in ice concentration retrieval introduced by the land impact on brightness temperatures in coastal regions. We addressed the difficulties arising when sea ice concentration in coastal areas is calculated using the NASA Team or the ASI algorithm. We shortly presented the basic principles of the NASA Team and the ASI algorithm, which served as exemplary fields of application.

A method was derived to extract the oceanic contribution to the measured brightness temperatures which can be inserted to these sea ice algorithms.

Because the environmental conditions in the brackish waters of the Baltic Sea differ significantly from the conditions encountered in the Arctic some variables used in the ice concentration algorithms had to be adjusted to applications in the less saline Baltic Sea. The ASI algorithm tiepoints needed as references for open water and complete ice coverage were determined analysing SSM/I data from the winter seasons 2008 and 2009. Furthermore, the thresholds for the weather filters used in both ice concentration algorithms considered in this study were adapted to Baltic conditions. Due to the sensitivity of ice concentration retrieval to the ice situation the thresholds were determined separately for freezing and melting conditions.

First applications to the Bothnian Bay's winter ice field in the Baltic sea showed a significant improvement of the ice concen- tration in coastal regions. We successfully removed the systematic assignment of non-zero ice concentration values to ice-free grid cells in the vicinity of land surfaces. However, the separation method caused the NASA Team algorithm to slightly underestimate the land-fast ice surrounding the island Holmön and in the Finnish archipelago off Vaasa.

We find it particularly encouraging that apart from this slight underestimation in case of the NASA Team algorithm the actually ice-covered areas in the land influenced regions are however recognized and assigned reasonable ice concentration values.

The ice edge was depicted realistically for both algorithms when the separation method was applied previous to ice concentration retrieval.

\section{Acknowledgments}

Enivsat ASAR data provision by ESA. SSM/I data have been obtained from Global Hydrology Resource Center (GHRC), Huntsville, AL, USA. MODIS data have been obtained from NASA Goddard Land Processes data archives (LAADS). This work has been funded by the German Science Foundation (DFG) via SFB512, Teilprojekt E5.

\section{References}

Bellerby, T., Taberner, M., Wilmshurst, A., Beaumont, M., Barrett, E., and co-authors. 1998. Retrieval of land and sea brightness temperatures from mixed coastal pixels in passive mircowave Data. IEEE Trans. Geosci. Remote Sens. 36, 1844-1851.

Bennartz, R. 1999. On the use of SSM/I measurements in coastal regions. J. Atmos. Oceanic Technol. 16, 417-431.

CAL/VAL 1998. DMSP Special Sensor Microwave/Imager Calibration/ Validation, NRL Final Report 2, Naval Research Laboratory, Washington, DC, 453 pp.

Cavalieri, D. J., Crawford, J. P., Drinkwater, M. R., Eppler, D. T., Farmer, L. D. and co-authors. 1991. Aircraft active and passive microwave validation of sea ice concentration from the Defense Meteorological Satellite Program Special Sensor Microwave Imager. J. Geophys. Res.-Oceans 96(C12), 21 989-22008.

Cavalieri, D. J., St. Germain, K. M. and Swift, C. T. 1995. Reduction of weather effects in the calculation of sea-ice concentration with DMSP SSM/I. J. Glaciol. 41(139), 455-464.

Drusch, M. 2006. Sea ice concentration analyses for the Baltic Sea and their impact on numerical weather prediction. J. Appl. Meteorol. Climatol. 45, No. 7, American Meteorological Society, 982-994.

Drusch, M., Wood, E. F. and Lindau, R. 1999. The impact of the SSM/I antenna gain function on land surface parameter retrieval. Geophys. Res. Lett. 26, 3481-3484.

Gloersen, P. and Cavalieri, D. J. 1986. Reduction of weather effects in the calculation of sea ice concentration from microwave radiances. J. Geophys. Res. 136(C3), 3913-3919.

Grandell, J. and Hallikainen, M. 1994. Modeling and retrieval of snow and sea ice characteristics in the frequency range 6 to $90 \mathrm{GHz}$. Final report. Report 21, Laboratory of Space Technology, Helsinki University of Technology, Finland. 
Hollinger, J. P. 1990. DMSP Special Sensor Microwave/Imager Calibration/Validation. Final Report, Vol. I., Naval Research Laboratory, Washington, DC.

Kaleschke, L., Lüpkes, C., Vihma, T., Haarpaintner, J., Bochert, A. and co-authors. 2001. SSM/I sea ice remote sensing for mesoscale ocean-atmosphere interaction analysis. Can. J. Remote Sens. 27(5), 526-537.

Leppäranta, M. and Myrberg, K. 2009. Physical Oceanography of the Baltic Sea. Springer-Verlag, Berlin, Germany.

Markus, T. and Cavalieri, D. J. 2000. An enhancement of the NASA Team sea ice algorithm. IEEE Trans. Geosci. Remote Sens. 38, 1387-1398.

Maslanik, J. A., Serreze, M. C. and Barry, R. G. 1996. Recent decreases in Arctic summer ice cover and linkages to atmospheric circulation anomalies. Geophys. Res. Lett. 23, 1677-1680.

NSIDC. 1996. Investigating mixed errors in SSM/I data processed with the AES/York sea ice concentration algorithm. NSIDC Notes Issue 18.
Parkinson, C., Comiso, J., Zwally, H., Cavalieri, D., Gloersen, P. and coauthors. 1987. Arctic sea ice, 1973-1976: Satellite passive-microwave observations, NASA SP-489, National Aeronautics and Space Administration, Washington, DC.

Shokr, M., Asmus, K. and Agnew, T. A. 2009. Microwave emission observations from artificial thin sea ice: the ice-tank experiment. IEEE Trans. Geosci. Remote Sens. 47(47), 325-338.

Spreen, G., Kaleschke, L. and Heygster, G. 2008. Sea ice remote sensing using AMSR-E 89-GHz channels. J. Geophys. Res. 113, 3481-3484.

Steffen, K. and Schweiger, A. 1991. NASA Team algorithm for sea ice concentration retrieval from DMSP SSM/I: Comparison with Landsat satellite imagery. J. Geophys. Res. 96, 21 971-21 987.

Swift, C. T., Cavalieri and D. J. 1985. Passive microwave remote sensing for sea ice research. EOS. 66(49), 1210-1212.

Wessel, P. and Smith, W. H. F. 1996. A global, self-consistent, hierarchical, high-resolution shoreline database. J. Geophys. Res.-Solid Earth 101(B4). 